Research Paper

\title{
CXCR4/CXCR7/CXCL12-Axis in Follicular Thyroid Carcinoma
}

\author{
Thomas Artur Werner1 ${ }^{1}$, Christina Maria Forster1, Levent Dizdar1, Pablo Emilio Verde2, Katharina Raba ${ }^{3}$, \\ Matthias Schott ${ }^{4}$, Wolfram Trudo Knoefel ${ }^{1}$, Andreas Krieg ${ }^{1 凶}$ \\ 1. Department of Surgery (A), Heinrich-Heine-University and University Hospital Duesseldorf, Moorenstr. 5, 40225 Duesseldorf, Germany \\ 2. Coordination Centre for Clinical Trials, Heinrich-Heine-University and University Hospital Duesseldorf, Moorenstr. 5, 40225 Duesseldorf, Germany \\ 3. Institute for Transplantation Diagnostics and Cell Therapeutics, Heinrich-Heine-University and University Hospital Duesseldorf, Moorenstr. 5, 40225, \\ Duesseldorf, Germany \\ 4. Division of Endocrinology, Heinrich-Heine-University and University Hospital Duesseldorf, Moorenstr. 5, 40225 Duesseldorf, Germany \\ $\triangle$ Corresponding author: Andreas Krieg, Department of Surgery (A), Heinrich-Heine-University and University Hospital Duesseldorf, Moorenstr. 5, Bldg. \\ 12.46, 40225 Duesseldorf, Germany; Phone: + 4921181 19251; Fax: + 4921181 19205; Email: andreas.krieg@med.uni-duesseldorf.de \\ (c) Ivyspring International Publisher. This is an open access article distributed under the terms of the Creative Commons Attribution (CC BY-NC) license \\ (https://creativecommons.org/licenses/by-nc/4.0/). See http://ivyspring.com/terms for full terms and conditions.
}

Received: 2017.09.27; Accepted: 2018.02.13; Published: 2018.02.27

\begin{abstract}
Background: Follicular thyroid carcinoma's (FTC) often benign course is partially due to adjuvant radioactive iodine (RAI) treatment. However, once the tumour has spread and fails to retain RAI, the therapeutic options are limited and the outcome is poor. In this subset of patients, the identification of novel druggable biomarkers appears invaluable. Here, we investigated the stage dependent expression and functional role of the C-X-C chemokine receptors type 4 and 7 (CXCR4/7) in FTC.

Methods: CXCR4/7 expression was examined in 44 FTC and corresponding non-neoplastic thyroid specimens as well as 10 FTC distant metastases and 18 follicular adenomas using tissue microarray technology. Expression levels were correlated with clinicopathological variables as well as overall and recurrence free survival. Changes regarding cell cycle activation, tumour cell invasiveness and mRNA expression of genes related to epithelial-mesenchymal transition (EMT) were investigated after treatment with recombinant human SDFla/CXCL12 (rh-SDFla) and CXCR4 antagonists AMD3100 and WZ811.

Results: CXCR4/7 expression was associated with large tumour size, advanced UICC stage as well as shorter overall and recurrence free survival. CXCR4 was significantly higher expressed in distant metastases than in primary tumour cores. In addition, rh-SDFla induced invasive growth, cell cycle activation and EMT, while CXCR4 antagonists significantly reduced FTC invasiveness in vitro.

Conclusion: Here we provide first evidence of the biological importance of the CXCR4/CXCR7/CXCL12 axis in FTC. Our findings underscore the therapeutic potential of this chemokine receptor family in advanced FTC and offer new valuable insight into the oncogenesis of metastatic FTC.
\end{abstract}

Key words: CXCR4, CXCR7, CXCL12, FTC, metastasis

\section{Introduction}

Follicular thyroid carcinomas (FTC) are the second most common malignant tumour of the thyroid gland. They originate from the follicular cells and belong to the group of differentiated thyroid carcinomas (DTC). FTCs are characterised by an invasive growth pattern and the forming of early distant metastases, affecting especially lungs and bones ${ }^{1}$. While surgery can hold the cure in the early and localized stages of the disease, a large amount of patients already harbour distant metastases at the time of initial presentation ${ }^{2,3}$. For these patients, mere radical oncological surgery is insufficient. However, 
due to their differentiated nature, FTC cells regularly take part in iodine metabolism and are therefore susceptible to postoperative radioactive iodine (RAI) treatment. This circumstance is the primary determinant of FTC's often benign course apart from the possibility to resect the tumour completely. Even with distant metastases the overall survival rate remains at approximately $50 \%$ over five years 4 . However, once the tumour loses this ability and becomes non-RAI avid, the prognosis tumbles to a mere $19 \%$ overall survival over five years 5,6 .

Over the last couple of years, a new appraisal of the RAI refractory FTC has taken hold of the endocrine research community. Approximately one third of all patients with DTC and distant metastases lack RAI uptake, while once RAI positive tumours can turn RAI refractory during the course of the disease ${ }^{5-7}$. However, despite increasing research efforts in this particular field of oncology, thus far, no adequate substitute for RAI treatment exists.

Since the occurrence of distant metastases is the most relevant prognostic determinant of the future clinical outcome ${ }^{1,2}$, a more profound understanding of the molecular mechanics responsible for tumour cell dissemination appears invaluable in devising new treatment strategies. In this regard the CXCR4/ CXCR7/CXCL12-axis has raised considerable attention over the last years, proving an important cornerstone in the metastasizing process in a variety of malignant tumours ${ }^{8}$. C-X-C chemokine receptors type 4 and 7 (CXCR4, CXCR7) are G protein-coupled receptors, which are responsible for cell trafficking, tissue development and immune response. Whilst widely expressed during embryogenesis, CXCR4 is mostly absent in adult tissues under physiological conditions. However, during the malignant progression of a variety of epithelial tumours, CXCR4 becomes markedly overexpressed ${ }^{9}$. The predominant ligand to CXCR4 is the stromal cell-derived factor 1 (SDF1), also known as C-X-C motif chemokine 12 (CXCL12), which is a potent chemoattractant for CXCR4-expressing cells ${ }^{10-12}$. Interestingly, CXCL12 is highly expressed in common metastatic sites like liver, lung and bone marrow. This striking aspect of CXCL12 expression encourages the assumption that CXCL12 plays a crucial role in the homing of disseminated tumour cells to their respective distant metastatic sites. Besides chemotaxis, CXCL12 also causes the upregulation of cellular pathways that are associated with transendothelial migration, epithelial-mesenchymal transition (EMT), proliferation and the inhibition of apoptosis ${ }^{13}$.

The functional role of CXCR7 on the other hand is much more controversial. Whilst some studies have identified CXCR7 as a decoy receptor ${ }^{14}$, which does not activate G-protein coupled pathways, others consider CXCR7 a signal transducing receptor, which is activated through CXCL12-binding ${ }^{15}$. These observations have led to the idea that CXCR7 functions as a co-receptor to CXCR4, which increases CXCL12-mediate G-protein signalling ${ }^{16}$. Despite these controversies, increasing evidence of CXCR7 participation in tumour development, neovascularization and invasion has been accumulating ${ }^{17-19}$. Its overexpression in different types of malignant tumours has been well documented and its activation causes tumour cell proliferation, invasion and neo-angiogenesis ${ }^{13}$. Despite the mounting evidence of the central role of the CXCR4/CXCR7/CXCL12-axis in the regulation of tumour cells' invasiveness and colony formation, is its role in FTC yet unknown.

The aim of our study was to investigate the expression of CXCR4 and CXCR7 in FTC and to correlate their expression patterns with clinicopathological variables as well as further stratifying their biological role in vitro.

\section{Material and Methods}

\section{Patient selection}

Three sets of patients were included in this study. Firstly, all patients who underwent curative surgery for histologically confirmed FTC at the Department of Surgery (A), University Hospital Duesseldorf between 1992 and 2014 were evaluated for inclusion. Tissue specimens that offered only insufficient pathological reports, fragmentary clinical data, incomplete resection margins or not enough tumour material for subsequent analyses were excluded from this study. The clinicopathological data was obtained from our prospectively maintained clinical database. The data included age at first diagnosis, gender, date and type of surgery, affected lobe, TNM stage and overall as well as recurrence free survival. Overall survival was defined as time in months beginning from the date of surgery until death of any cause. Survivors lost to follow-up were censored at the respective time. The included tumour specimens were all staged according to the $8^{\text {th }}$ edition of the UICC classification ${ }^{20}$.

Secondly, tissue samples from 10 patients who underwent surgical resection of a distant metastasis of their FTC alone and, thirdly, 18 follicular thyroid adenomas were included. The same inclusion and exclusion criteria as stated above were applied.

The study was executed according to Good Clinical Practice, the Declaration of Helsinki and local rules as well as regulations of the country. In addition, it was approved by the institutional ethics committee of the Medical Faculty of the Heinrich Heine 
University, Duesseldorf (reference number: 3821).

\section{Tissue microarray and immunohistochemistry}

Formalin-fixed paraffin-embedded tissue samples were obtained from the Institute of Pathology, University Hospital Duesseldorf. All tissue samples were newly reviewed prior to this study and the diagnosis of FTC histologically confirmed. FTCs were defined as thyroid carcinomas with follicular differentiation in the absence of papillary nuclear features, with either capsular or vascular invasion. The construction of the tissue microarray, immunohistochemistry and protein expression analyses were performed as described previously ${ }^{21,22}$. The expression levels of CXCR4 and CXCR7 were scored by two independent investigators (TW, CF) using the immunoreactivity score (IRS) reported by Remmele ${ }^{23}$ in a blinded manner.

The immunohistochemistry was carried out using mouse monoclonal anti-CXCR4 (1:100 dilution; Abcam, Cambridge, UK) and rabbit polyclonal anti-CXCR7 (1:200 dilution; GeneTex, Irvine, CA, USA) as primary antibodies. Isotype control was performed using mouse IgG1k (MOPC-21; 1:50 dilution; Abcam, Cambridge, UK) and rabbit immunoglobulin fraction (Code X0903; 1:1000 dilution; Dako, Glostrup, Denmark). CXCR4 expressing tonsil tissue and CXCR7 expressing pancreatic adenocarcinoma served as positive controls. The prognostic power of CXCR4 and CXCR7 was assessed in accordance with the REporting recommendations for tumour MARKer prognostic studies (REMARK) ${ }^{24}$.

\section{Cell culture and reagents}

The human FTC cell line TT2609-C02 was obtained from the Leibniz Institute DSMZ-German Collection of Microorganisms and Cell Cultures (DSMZ no.: ACC 510). The cell line was cultivated in RPMI Media 1640 (Gibco Thermo Fisher, MA, USA), supplemented with $10 \%$ fetal bovine serum. This FTC cell line was cultivated from a local tumour recurrence of a secondary RAI refractory FTC, thus proving an excellent model for our experiments ${ }^{25}$. Authenticity of the cell line was verified by short tandem repeat genetic profiling as previously described $^{26}$. Specific CXCR4 antagonists plerixafor (AMD3100) and WZ811 were purchased from Selleck Chemicals (TX, USA), while recombinant human (rh) SDF1a was obtained from PeproTech (NJ, USA).

\section{Invasion assay}

Changes in cell invasiveness after treatment with different concentrations of rh-SDF1a, AMD3100, WZ811 were investigated using the BD Matrigel invasion chamber (BD Biosciences, Heidelberg,
Germany). FTC cells TT2609-C02 were serum starved $24 \mathrm{~h}$ prior to the experiment. Cells were then seeded at a density of $5 \times 10^{4}$ cells $/ \mathrm{ml}$ in serum-free medium, supplemented with $0.25 \%$ bovine serum albumin and increasing concentrations of SDF1a, AMD3100, WZ811 or vehicle control at equimolar concentrations. Conditioned Dulbecco's Modified Eagle Medium from CXCL12 expressing NIH3T3 fibroblasts was added to the lower chamber and served as chemoattractant ${ }^{27,28}$. After $24 \mathrm{~h}$ of incubation the noninvading cells were gently removed with a cottontipped swab. Invasive cells were fixated using methanol and stained with 4',6-diamidin-2-phenylindol (DAPI; $200 \mathrm{ng} / \mathrm{ml}$ ). Invaded cells were counted in five visual fields of three membranes under a fluorescence microscope at 200x magnification (Zeiss Axioplan 2, Carl Zeiss, Göttingen, Germany).

\section{FACS cell cycle analysis}

FTC cells were incubated with increasing concentrations of rh-SDF1a, AMD3100, WZ811 or vehicle control at equimolar concentrations for $48 \mathrm{~h}$. Cell cycle analyses were performed by propidium iodide (PI) staining. Cells were trypsinized and washed in cold PBS and resuspended in $80 \%$ ethanol. After 2 hours, cells were washed in PBS and incubated with RNAse A $(100 \mu \mathrm{g} / \mathrm{ml})$ and PI $(50 \mu \mathrm{g} / \mathrm{ml})$ for 30 minutes at $37^{\circ} \mathrm{C}$. The analyses were performed by fluorescence-activated cell sorting (FACS) using a BD FACSCanto ${ }^{\mathrm{TM}}$ II (BD Biosciences, San Jose, CA, USA).

\section{Western Blot analysis}

Cells were harvested using ice-cold RIPA buffer. Total protein concentrations were determined using a BioPhotometer ${ }^{\circledR}$ according to Bradford (Eppendorf, Hamburg, Germany). Protein separation was achieved by SDS-polyacrylamid gel electrophoresis and subsequent transfer onto a nitrocellulose membrane. Antibody-detection was performed overnight using the following primary antibodies: mouse monoclonal anti-CXCR4 (1:100 dilution; Abcam, Cambridge, UK), rabbit polyclonal anti-CXCR7 (1:200 dilution; GeneTex, Irvine, CA, USA).

\section{Immunocytochemistry}

Cells were cultivated in 6-well chamber slides and fixated with ice-cold methanol. After treatment with $0.5 \%$ Triton the cells were blocked with $20 \% \mathrm{AB}$ serum and incubated with their respective primary antibody and isotype control (mouse monoclonal anti-CXCR4, 1:250 dilution; Abcam, Cambridge, UK; rabbit polyclonal anti-CXCR7, 1:500 dilution; GeneTex, Irvine, CA, USA; mouse IgG1k (MOPC-21); $2 \mu \mathrm{g} / \mathrm{ml}$; Abcam, Cambridge, UK; rabbit immunoglobulin fraction (Code X0903) $2 \mu \mathrm{g} / \mathrm{ml}$; Dako, Glostrup, Denmark). After 45 minutes of 
incubation, cells were washed and incubated with anti-mouse- or anti-rabbit-Alexa Fluor ${ }^{\circledR} \quad 488 \quad(10$ $\mu \mathrm{g} / \mathrm{ml}$; Thermo Fisher, MA, USA), respectively. Nuclear staining was carried out with DAPI (200 $\mathrm{ng} / \mathrm{ml})$. After fixating the cells with $1 \%$ PFA, visualization was achieved using a fluorescence microscope at 400x magnification (Zeiss Axioplan 2, Carl Zeiss, Göttingen, Germany).

\section{RT-PCR}

The RNeasy Mini Kit was used to extract total RNA from treated cells according to the manufacturer's protocol (Qiagen, Hilden, Germany). Complementary DNA was subsequently synthesized from $2 \mu \mathrm{g}$ of total RNA, using the qScript cDNA Synthesis Kit (QuantaBio, MA, USA) following the manufacturer's instructions. After adjusting the cDNA to a final concentration of $1 \mathrm{ng} / \mu \mathrm{l}, 2 \mu \mathrm{l}$ of cDNA were mixed with $10 \mu \mathrm{l}$ PerfeCTa ${ }^{\circledR}$ FastMix $^{\circledR}$ II (QuantaBio, MA, USA), $0.2 \mu$ l probe (100 nM; Roche, Mannheim, Germany) and $0.2 \mu \mathrm{l}$ of forward and reverse primer (200 $\mathrm{nM}$ each) for quantitative real-time-PCR. The PCR slides were constructed as triplicates. The primers used are listed in Supplementary Table S1. We used Glyceraldehyde3-phosphate dehydrogenase (GAPDH) as internal reference gene. PCR runs were performed on the DyadDisciple Chromo 4 (BioRad) with the following set-up: $95^{\circ} \mathrm{C}$ for 2 minutes, followed by 40 cycles of denaturation at $95{ }^{\circ} \mathrm{C}$ for 10 seconds and annealing and extension at $60{ }^{\circ} \mathrm{C}$ for 30 seconds. Gene expression was quantified according to the $2^{-\Delta \Delta C T}$ method ${ }^{29}$.

\section{Statistical analysis}

Regarding the evaluation of a possible association between the expression of CXCR4/7 and different clinicopathological markers, we employed the non-parametric Mann-Whitney U test. To further validate the data, we grouped the samples according to their IRS for CXCR4 and CXCR7 into high (IRS $\geq$ median) and low expression (IRS < median) and analysed the data using the Fisher's exact test.

The log-rank (Mantel Cox) test was used for univariate survival analyses. For multivariate analysis, a Cox regression analysis was performed to estimate hazard ratios (HR) with $95 \%$ confidence intervals $(\mathrm{CI})$ for all investigated variables. Afterwards, a search for the best statistical model was performed employing a stepwise variable selection procedure based on the Akaike Information Criterion (AIC). Additionally, we performed a survival regression tree analysis to identify subgroups of patients with very high-risk of death. This technique combines an algorithm for recursive partitioning with permutation tests. The resulting partitioning is graphically displayed as a classification tree, each node displaying an optimal cut-off point for the respective continuous co-variable.

Cell culture experiments were performed at least thrice and evaluated for statistical significance using the nonparametric Mann-Whitney U test.

Statistical analyses were conducted using GraphPad Prism (Version 6, GraphPad Software, San Diego, California, USA) and the Statistical Software R version 3.1.0 (R Development Core Team, 2014). A p-value $<0.05$ was considered statistically significant.

\section{Results}

\section{Patients and outcome}

A total of 44 patients were included in this study. The clinicopathological characteristics are summarized in Table 1. Two tissue discs were lost during the staining procedure for CXCR4, while 4 discs were lost for CXCR7. Overall, ten of the 44 patients could not be included in the survival analysis due to loss of follow-up. The median overall survival was 47 months (range 0-197 months) with a median follow-up of 62 month (range 0-197 months) based on the reverse Kaplan-Meier procedure. During the observation period 9 patients died. The last follow-up was conducted in December 2015.

Table 1: Patient characteristics $(n=44)$

\begin{tabular}{lc}
\hline Variables & No. of patients (\%) \\
\hline Total & 44 \\
Age & \\
Median (range); years & $59(17-85)$ \\
Gender & \\
Male & $19(43)$ \\
Female & $25(57)$ \\
Type of surgery & \\
Hemithyroidectomy & $3(7)$ \\
with unilateral ND & $1(2)$ \\
Subtotal thyroidectomy & $11(25)$ \\
with unilateral ND & $4(9)$ \\
with central LND & $1(2)$ \\
Total thyroidectomy & $7(16)$ \\
with central LND & $4(9)$ \\
with unilateral ND & $2(5)$ \\
with bilateral ND & $11(25)$ \\
Tumour stage & \\
T1/2 & $24(55)$ \\
T3/4 & $20(45)$ \\
Lymph node metastasis & \\
N0 & $41(93)$ \\
N1a/b & $3(7)$ \\
Distant metastasis & \\
M0 & $38(86)$ \\
M1 & $6(14)$ \\
UICC stage & \\
UICC I/II & $22(50)$ \\
UICC III/IV & $22(50)$ \\
\hline Abbreviations: ND: neck dissection; LND: lymph node dissection; UICC: Union \\
internationale contre le cancer. &
\end{tabular}




\section{CXCR4 and CXCR7 expression in FTC and non-neoplastic thyroid tissue}

CXCR4 and CXCR7 were significantly higher expressed in FTC tissue specimens, when compared to their corresponding non-neoplastic thyroid tissue samples (CXCR4: $p$ < 0.001; CXCR7: $\mathrm{p}<0.001$; Figure 1A-C). However, while CXCR4 demonstrated a moderate expression in normal thyroid tissue, all non-neoplastic thyroid tissue samples stained negatively for CXCR7. Next, we compared the expression of the two chemokine receptors in adenomas of the thyroid gland and non-neoplastic thyroid tissue specimens as well as FTC samples, respectively. CXCR4 demonstrated a significant higher expression in adenoma tissues when compared to non-neoplastic thyroid tissue specimens $(p<0.001$; Figure 1B). The expression level of CXCR4 adenoma tissue specimens was as high as in FTC tissue specimens (Figure 1B). Regarding CXCR7, the adenoma tissue specimens also exhibited a significant higher expression then their non-neoplastic counterparts $(p=0.0046$; Figure $1 C)$ but the expression level was also significantly lower than in the FTC tissue specimens ( $p=0.0120$; Figure 1C).

\section{Distant metastases exhibit significant higher expression levels of CXCR4}

Since most of our patients included in the TMA did not harbour distant metastases at the time of treatment (Table 1), we investigated the expression levels of the two chemokine receptors in a second set of patients, where only tissue samples of distant metastases were available. Interestingly, when comparing these two groups with regard to their CXCR4 expression, we found the disseminated tumour cells demonstrating a significantly higher expression of CXCR4 than the primary tumour cells ( $\mathrm{p}$ $=0.0379$; Figure 1B). This difference in expression, however, was not evident for CXCR7 (Figure 1C).
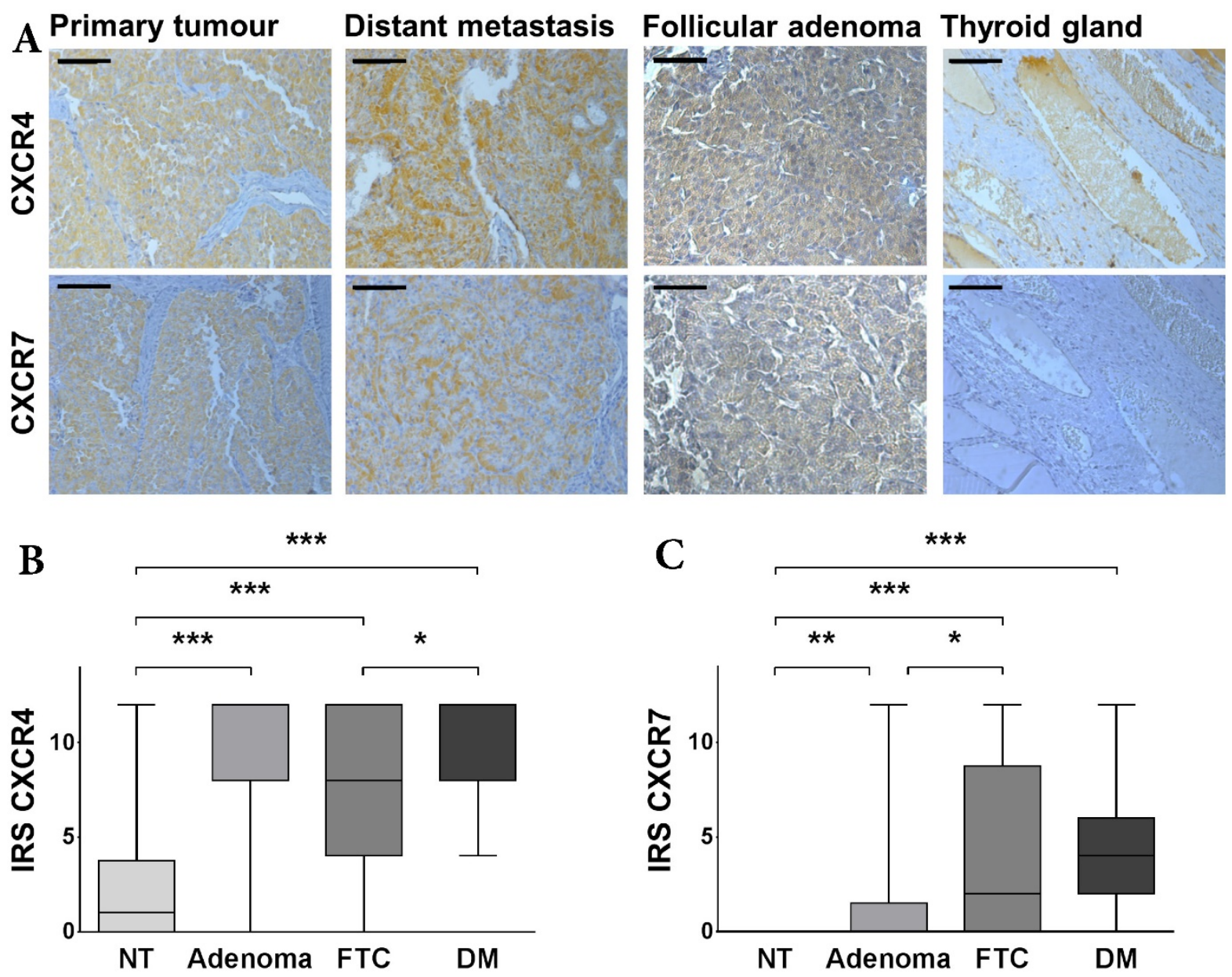

Figure 1: Expression of CXCR4 and CXCR7 in FTC. (A) Representative tissue samples of FTC, distant metastasis, follicular adenoma and non-neoplastic thyroid tissue, immunohistochemically stained for CXCR4 and CXCR7. All samples were classified as strong expression of the given marker. The bar in the top left corner indicates $50 \mu \mathrm{m}$. (B/C) Boxplots display the expression levels of CXCR4 and CXCR7 in non-neoplastic thyroid tissue specimens, follicular adenomas as well as FTC and distant metastases according to the IRS. Boxplots illustrate the median IRS with the upper and lower quartile, as well as maximum and minimum of the given marker. Expression levels of CXCR4 and CXCR7 in the different tissue specimens were compared between groups using the Wilcoxon matched-pairs signed rank test and the non-parametric Mann-Whitney $U$ test as indicated. Bars mark the respective pairs. IRS: immunoreactivity score; $C X C R 4 / 7$ : C-X-C chemokine receptor type 4/7; NT: non-neoplastic thyroid gland; FTC: follicular thyroid carcinoma; DM: distant metastases; ${ }^{*} p<0.05 ; * * p<0.01 ; * * * p<0.001$. 
Table 2: Correlation between CXCR4 or CXCR7 and clinicopathological parameters in FTC

\begin{tabular}{|c|c|c|c|c|c|c|}
\hline \multirow[t]{2}{*}{ Variables } & \multicolumn{3}{|c|}{ CXCR4 expression } & \multicolumn{3}{|c|}{ CXCR7 expression } \\
\hline & Low, $\mathrm{n}=18(\%)$ & High, $n=24(\%)$ & $p$-value & Low, $n=15(\%)$ & High, $\mathbf{n}=25(\%)$ & $p$-value \\
\hline Age, mean \pm s.d. & $52.6 \pm 17.3$ & $58.9 \pm 16.9$ & 0.756 & $56.2 \pm 18.9$ & $55.8 \pm 15.4$ & 1 \\
\hline \multicolumn{7}{|l|}{ Gender } \\
\hline Male & $5(28)$ & $13(54)$ & & $3(20)$ & $12(40)$ & \\
\hline Female & $13(72)$ & $11(46)$ & 0.120 & $12(80)$ & $13(60)$ & 0.101 \\
\hline \multicolumn{7}{|l|}{ Tumour stage } \\
\hline $\mathrm{T} 1 / 2$ & $14(28)$ & $9(38)$ & & $12(20)$ & $10(40)$ & \\
\hline $\mathrm{T} 3 / 4$ & $4(72)$ & $15(62)$ & 0.013 & $3(80)$ & $15(60)$ & 0.022 \\
\hline \multicolumn{7}{|c|}{ Lymph node metastasis } \\
\hline N0 & $17(94)$ & $22(92)$ & & $14(93)$ & $23(92)$ & \\
\hline $\mathrm{N} 1 \mathrm{a} / \mathrm{b}$ & $1(6)$ & $2(8)$ & 1 & $1(7)$ & $2(8)$ & 1 \\
\hline \multicolumn{7}{|c|}{ Distant metastasis } \\
\hline M0 & $16(89)$ & $20(83)$ & & $13(87)$ & $21(84)$ & \\
\hline M1 & $2(11)$ & $4(17)$ & 0.685 & $2(3)$ & $4(16)$ & 1 \\
\hline \multicolumn{7}{|l|}{ UICC stage } \\
\hline UICC I/II & $13(72)$ & $8(33)$ & & $11(73)$ & $9(36)$ & \\
\hline UICC III/IV & $5(28)$ & $16(67)$ & 0.0278 & $4(27)$ & $16(64)$ & 0.0484 \\
\hline \multicolumn{7}{|l|}{ Invasiveness } \\
\hline minimally & $11(61)$ & $9(38)$ & & $10(67)$ & $10(40)$ & \\
\hline \multirow[t]{2}{*}{ widely } & $7(39)$ & $15(62)$ & 0.212 & $5(33)$ & $15(60)$ & 0.191 \\
\hline & Low, $\mathbf{n} 10=(\%)$ & High, $n=15(\%)$ & $p$-value & Low, $\mathrm{n}=8(\%)$ & High, $n=16(\%)$ & $p$-value \\
\hline \multicolumn{7}{|l|}{ Relapse } \\
\hline No relapse & 9 & 7 & & 8 & 8 & \\
\hline Relapse & 1 & 8 & 0.0405 & 0 & 8 & 0.022 \\
\hline
\end{tabular}

Abbreviations: FTC: follicular thyroid carcinoma; CXCR4/7: C-X-C chemokine receptor type 4/7; UICC = Union internationale contre le cancer.

\section{CXCR4 and CXCR7 expression correlates with advanced tumour stage}

To investigate a potential association between the two chemokine receptors and different clinicopathological parameters, we compared the IRS across groups for each clinicopathological variable. CXCR4 and CXCR7 demonstrated a strong association with larger tumour sizes, exhibiting significantly higher expression in T3/4 tumours than compared to T1/2 tumours (CXCR4: $p=0.0361$; CXCR7: $p=0.0015$; Figure 2A/B). Similarly, CXCR4 and CXCR7 were significantly higher expressed in the advanced UICC III/IV tumour stages (CXCR4: $p=$ 0.0288; CXCR7: $p=0.0071$; Figure 2A/B). These observations were further verified by categorising the data with regard to their respective chemokine receptor expression into high ( $\geq$ median) and low (< median) and comparing the groups with the Fisher's exact test (Table 2).

\section{CXCR4 and CXCR7 expression are negative prognostic markers in FTC}

In the univariate survival analysis, a high CXCR4 expression was associated with a significantly worse prognosis (HR: 5.846; 95 \% CI: 1.442 - 23.69; p = 0.0134; Figure 2C, Table 3A) and significantly shorter recurrence free survival (HR: 4.146; 95 \% CI: 1.005 17.10; $p=0.0492$; Figure 2E). Similarly, patients with a high CXCR7 expression had a significantly higher

chance of death (HR: 4.553; 95 \% CI: 1.124 - 18.44; $\mathrm{p}=$ 0.0337; Figure 2D; Table 3A) and a significantly higher risk of recurrence (HR: 5.218; CI 95 \%: 1.106 - 24.63; p $=0.0369$; Figure $2 \mathrm{~F}$ ). In addition, patients with widely invasive FTC had a significantly shorter overall survival, than patients with minimally invasive FTC (HR: 5.164; 95 \% CI: 1.397 - 19.09; $p=0.0142$; Table 3A).

Table 3: Overall survival analysis

\begin{tabular}{|c|c|c|c|}
\hline \multicolumn{4}{|c|}{ A: Univariate survival analysis } \\
\hline Variables & HR & CI (lower-upper $95 \%$ ) & $p$-value \\
\hline Age at first diagnosis & 1.814 & $0.487-6.765$ & 0.375 \\
\hline Sex & 0.485 & $0.131-1.808$ & 0.281 \\
\hline Invasiveness & 5.164 & $1.397-19.09$ & 0.0142 \\
\hline UICC I/II vs. UICC III/IV & 2.503 & $0.670-9.358$ & 0.173 \\
\hline CXCR4 expression & 5.846 & $1.442-23.69$ & 0.0134 \\
\hline CXCR7 expression & 4.553 & $1.124-18.44$ & 0.0337 \\
\hline \multicolumn{4}{|c|}{ B: Multivariate survival analysis; AIC $=58$} \\
\hline Variables & HR & CI (lower-upper $95 \%$ ) & $p$-value \\
\hline Age at first diagnosis & 1.066 & $1.016-1.120$ & 0.0098 \\
\hline Sex & 1.236 & $0.292-5.241$ & 0.774 \\
\hline Invasiveness & 9.173 & $1.055-79.760$ & 0.0446 \\
\hline UICC I/II vs. UICC III/IV & 0.267 & $0.023-3.084$ & 0.290 \\
\hline CXCR4 expression & 1.183 & $0.912-1.535$ & 0.204 \\
\hline CXCR7 expression & 1.133 & $0.901-1.424$ & 0.286 \\
\hline \multicolumn{4}{|c|}{ C: Multivariate survival analysis after stepwise variable selection; AIC $=51$} \\
\hline Variables & HR & CI (lower-upper $95 \%$ ) & $p$-value \\
\hline Invasiveness & 6.117 & $1.008-1.106$ & 0.0206 \\
\hline CXCR4 expression & 1.280 & $0.9942-1.649$ & 0.055 \\
\hline
\end{tabular}


A

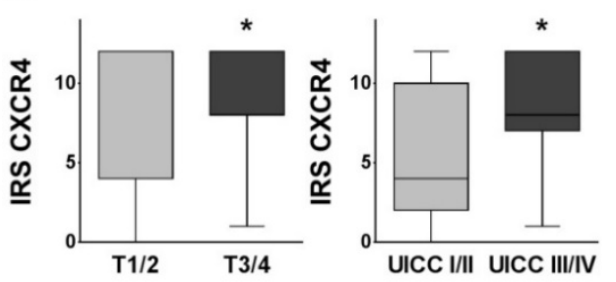

C

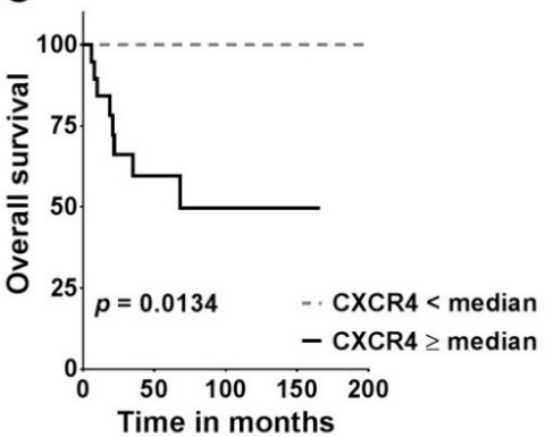

E

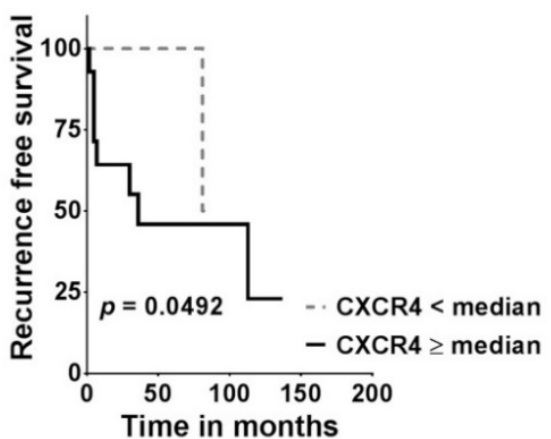

G

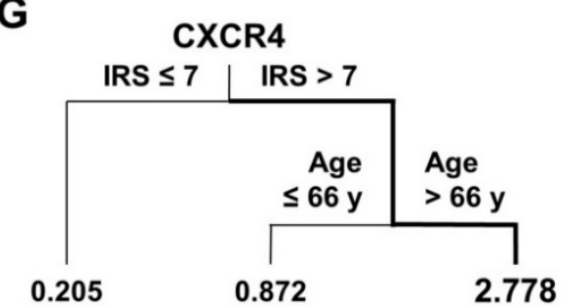

B

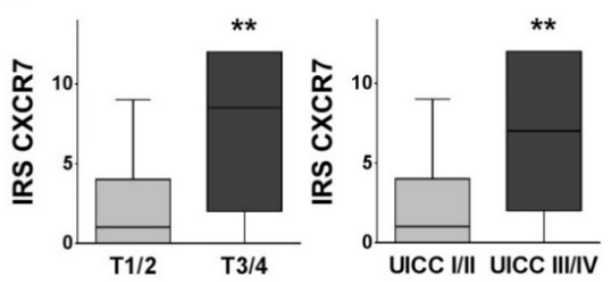

D

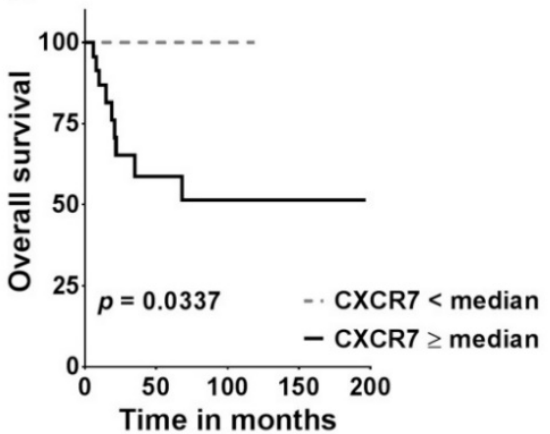

$\mathbf{F}$

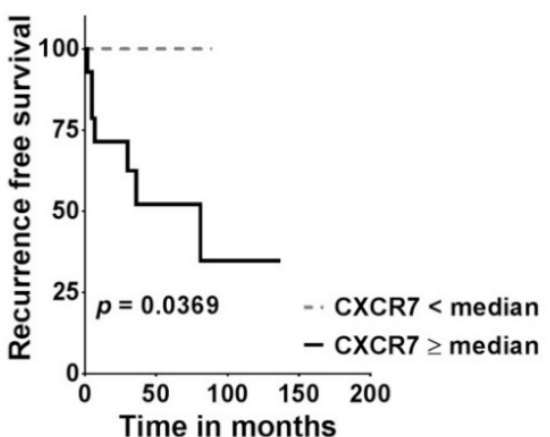

Figure 2: Association between CXCR4/7 expression and clinicopathological variables as well as overall and recurrence free survival. (A, B) Boxplots display the median IRS with the upper and lower quartile, as well as maximum and minimum for CXCR4 and CXCR7 in the primary tumour core grouped according to tumour size and UICC stage. CXCR4/7 expression levels were compared across groups employing the non-parametric Mann-Whitney U test. (C-F) For the overall and recurrence free survival analysis, patients were grouped according to their CXCR4/7 expression in their primary tumour cores into high $\geq$ median and low <median and groups were compared using the log-rank (Mantel Cox) test. (G) The survival regression tree analysis displays subgroups of patients with very high risk of death. Each junction represents a decision point with an optimal cut-off value for the respective variable and the numbers under the vertical lines represent hazard ratios. IRS: immunoreactivity score; CXCR4/7: C-X-C chemokine receptor type 4/7; UICC: Union internationale contre le cancer; ${ }^{*} p<0.05$; ${ }^{* *} p<0.01$ ).

In the multivariate analysis including all variables only the invasiveness of the tumour (HR: 9.173; $95 \%$ CI: $1.055-79.760 ; p=0.0446)$ and an advanced age at the time of first diagnosis (HR: 1.066; $95 \%$ CI: $1.016-1.120 ; \mathrm{p}=0.0098)$ proved to be independent negative prognostic markers in our cohort of patients (Table 3B). The subsequent variable selection procedure based on the AIC reconfirmed invasiveness as the primary determinant of overall survival (HR: 6.117; 95 \% CI: 1.008 - 1.106; $p=0.0206$;
Table 3C). Interestingly however, in this search for the best model, CXCR4 expression almost reached statistical significance as an independent negative prognostic marker in FTC (HR: 1.280; 95 \% CI: 0.9942 1.649; $p=0.055$; Table 3C). Of note, the AIC decreased during this stepwise variable selection procedure by 7 units, indicating a better goodness of fit of the selection model and its higher relevance in our set of data $^{30}$. Due to the similarity in events between $\mathrm{T}$ and UICC stage it was not possible to compute a 
multivariate analysis with both variables included. Here we decided on UICC stage because of its broader representation of the tumours biology, i.e. including $\mathrm{N}$ and $\mathrm{M}$ stage. Similarly, for recurrence free survival, due to the small number of events, the computation of a multivariate analysis was not feasible.

In addition, the survival regression tree analysis identified the constellation of a high CXCR4 IRS in the primary tumour (IRS $>7$ ) together with an advanced age at the time of first diagnosis (Age >66y) as the most unfavourable combination, with a 2.778 times higher likelihood of death (Figure 2G). The regression tree analysis also offers a hierarchical structure, indicating CXCR4 as the more dominant of the two markers.

\section{Rh-SDF la stimulates invasive growth in FTC cell line}

Human FTC cell line TT2609-C02 expresses both chemokine receptors CXCR4 and CXCR7. Both receptors could be detected in immunocytochemistry and western blot analyses (Figure 3A/B). To investigate their functional implications in FTC, we incubated FTC cell line TT2609-C02 with increasing concentrations of chemokine receptor agonist rh-SDF1a and quantified changes in invasiveness. The treatment with rh-SDF1a caused a significant increase in the number of invading cells (10 nM rh-SDF1a: $\mathrm{p}=$ 0.0252; 100 nM rh-SDF1a: $p=0.0047$; Figure 4A/B). Notably, the cells responded in a dose-dependent manner, with $100 \mathrm{nM}$ rh-SDF1a causing significantly more cells to invade into the Matrigel than cells incubated with $10 \mathrm{nM}$ rh-SDF1a $(\mathrm{p}=0.0092$; Figure 4B).

To further investigate the receptors' respective roles for invasiveness, we treated the cells with different concentrations of the well-characterized
CXCR4 antagonists AMD3100 and WZ811. Both compounds demonstrated a comparable faculty in preventing cells from invasive growth. AMD3100 reduced the number of invading cells to $35 \%$ of the control-treated cells for both $1 \mu \mathrm{M}$ and $100 \mathrm{nM}$ concentrations ( $p$ 0.001; Figure 4A/B). Similarly, the incubation with WZ811 caused a decrease of invading cells by approximately $75 \%$ ( $<<0.001$; Figure 4A/B). Notably, the co-incubation with rh-SDF1a could not overcome the inhibitory effect of the respective CXCR4-antagonist and resulted in the same number of invading cells co-incubated with vehicle-control (data not shown).

\section{Rh-SDF l $\alpha$ causes cell cycle activation and EMT}

Treatment of TT2609-C02 with rh-SDF1a resulted in significant changes in the respective cell cycle phases. The incubation with rh-SDF1a caused a significant decrease in cells in the G1 phase, whilst at higher concentrations of rh-SDF1a significantly more cells entered the $S$ and G2/M phase (Figure $4 \mathrm{C}$ ).

To investigate a possible association between rh-SDF1a treatment and the induction of EMT, we next evaluated changes in RNA expression of genes known to be involved in tumour cell invasion and EMT. Using qRT-PCR, we quantified changes in gene expression of bone marrow stromal cell antigen 2 (BST2), fibroblast growth factor 9 (FGF9), Twist homolog 1 (TWIST1), Snail family transcriptional repressor 1 (SNAI1) as well as E-cadherin and Vimentin. The incubation with rh-SDF1a caused a significant increase in the expression of BST2, FGF9, TWIST1, SNAI1 and Vimentin $(\mathrm{p}<0.01)$, whilst the expression of E-cadherin was markedly reduced $(\mathrm{p}<$ 0.001; Figure 4D).
A

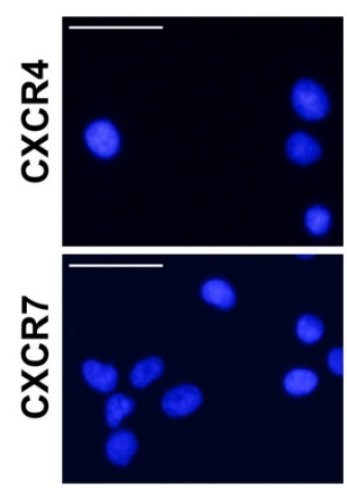

CXCR-AB

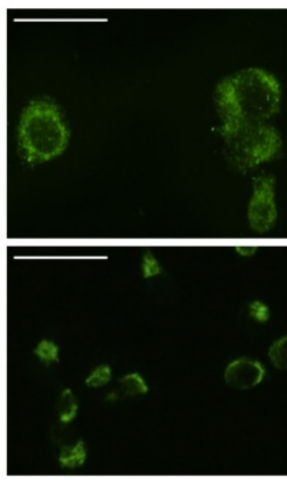

Overlay

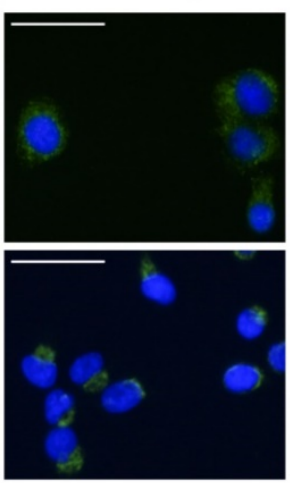

Control

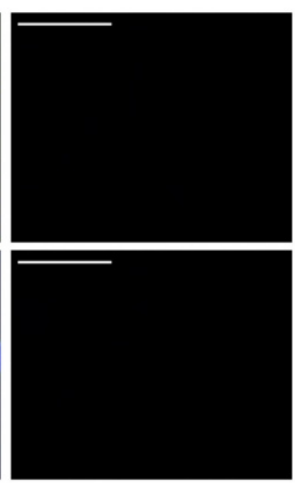

B

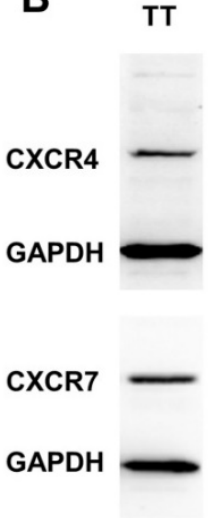

Figure 3: Expression levels of CXCR4 and CXCR7 in FTC cell line TT2609-C02. (A) FTC cell line TT2609-C02 exhibits both chemokine receptors CXCR4 and CXCR7. Immunocytochemical staining of CXCR4 and CXCR7 was conducted by using Alexa Fluor 848 as secondary antibody and DAPI for visualization of the nucleus. Compositions of both images are illustrated as overlays. Isotype controls were used to confirm antibody specificity (Control). Images were captured using a fluorescence microscope at 400x magnification. Bar at the top left corner indicates $50 \mu \mathrm{m}$. (B) FTC cell line TT2609-C02 demonstrates a strong protein expression of both chemokine receptors as displayed in the western blot analysis. GAPDH served as loading control. CXCR4/7: C-X-C chemokine receptor type 4/7; DAPI: 4',6-diamidin-2-phenylindol; GAPDH: Glyceraldehyde-3-phosphate dehydrogenase. 
A

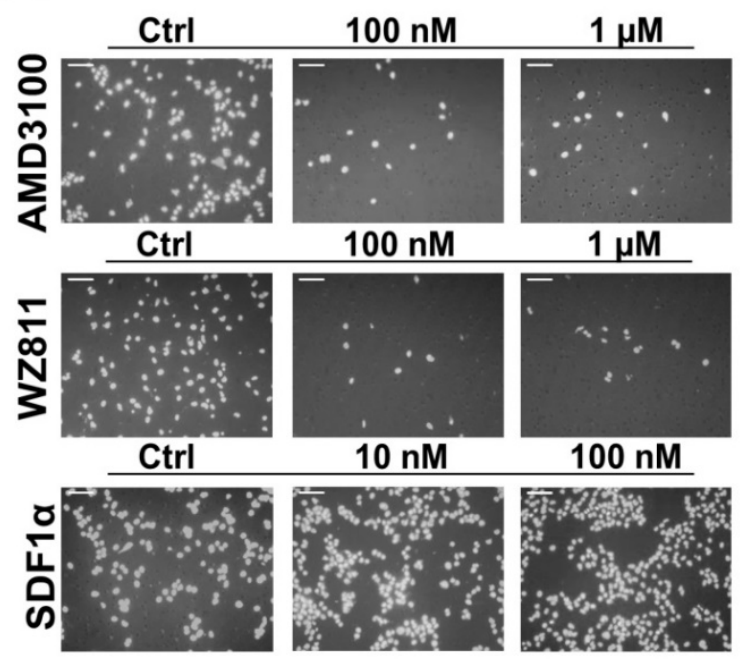

C
B

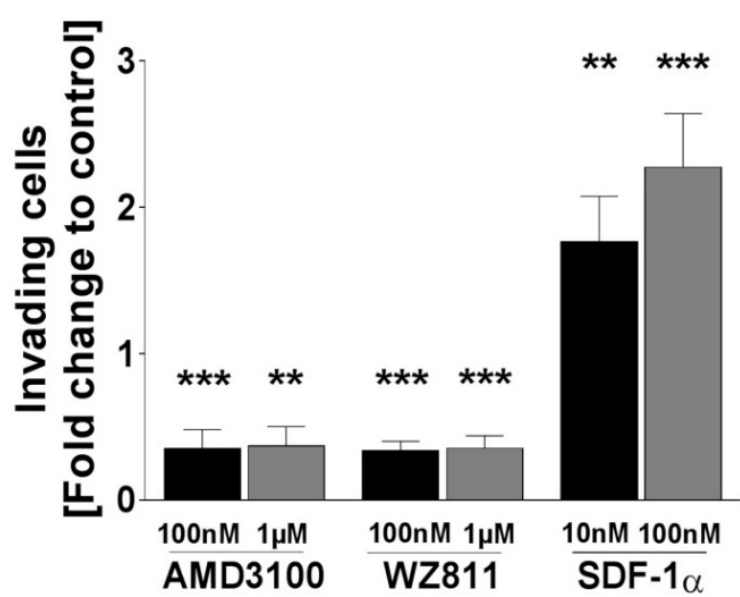

D

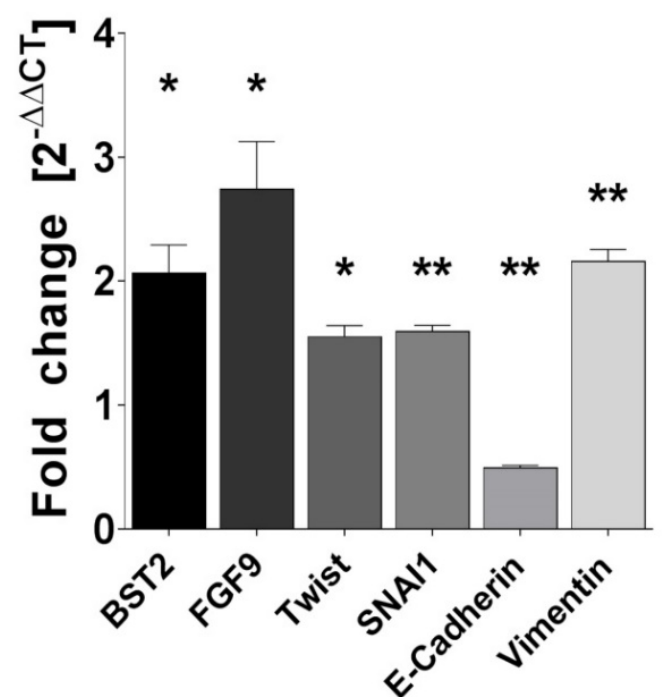

Figure 4: Rh-SDF l $\alpha$ induces tumour cell invasion, cell cycle activation and EMT. (A) Pictures display representative results of the change of invading cells after incubation of human FTC cell line TT2609-C02 with different concentrations of CXCR4 antagonists AMD3100 and WZ811 as well as rh-SDF1 $\alpha$ as receptor agonist. Cells were visualized by DAPI staining. (B) Invading cells were counted in five visual fields of at least three different membranes. Differences after treatment are illustrated as fold change to control. (C) FACS after PI staining revealed cell cycle changes in the respective cell populations after rh-SDFl $\alpha$ treatment. Cells are grouped according to their specific cell cycle phase. (D) After incubation with rh-SDFIa, mRNA expression changes of genes associated with EMT were investigated using qRT-PCR. GAPDH functioned as housekeeping gene. The 2- $\triangle \Delta C T$ method was employed to estimate fold changes to control treated cells. Plots display the mean +SEM. Numerical data were analysed using the non-parametric Mann-Whitney $U$ test. Ctrl: control; ${ }^{*} p<0.05 ; *_{*} p<0.01 ; * * * p<0.001$.

\section{Discussion}

FTCs account for approximately $15 \%$ of all thyroid malignancies ${ }^{1}$. Even though surgery can be curative at the early stages of the disease, FTCs invasive nature and haematogenous spread leave patients in dire need for adjuvant therapeutic strategies. Their generally favourable outcome can mainly be attributed to the tumour's capacity to retain $\mathrm{RAI}^{1,2}$. However, once the tumour fails to adequately retain RAI and becomes refractory to this targeted therapy, no comparable second line therapy is at hand to markedly influence the course of the disease ${ }^{7}$.
The chemokine receptors CXCR4 and CXCR7 have been associated as prognostic relevant biomarkers with different tumour entities. Over the last years even an ample body of scientific data regarding their role in papillary thyroid carcinoma (PTC) has emerged ${ }^{31-35}$. As a result, the CXCR4/ CXCR7/CXCL12 axis has gained considerable fame as a cornerstone in tumour cell invasiveness, cancer cell homing and the forming of distant metastases ${ }^{13,15}$. Nonetheless, little to none is known about its role in FTC. Thus far, only one study performed by Zhu et al. investigated the expression of CXCR4/7 in 10 FTC and follicular thyroid adenoma tissue specimens. 
However, besides the immunohistochemical staining procedure no subsequent analyses of possible correlations with clinicopathological variables or functional in vitro experiments regarding FTC were performed $^{34}$. Therefore, to our knowledge, ours is the first study that examined the stage dependent expression of CXCR4 and CXCR7 in FTC and further assessed their functional implications in vitro.

In our TMA analyses we found a significantly higher expression of the two chemokine receptors in the FTC tissue specimens when compared to their corresponding non-neoplastic thyroid tissue samples. Interestingly, already benign neoplastic lesions of the thyroid gland expressed significantly higher CXCR4 and CXCR7 IRS than their healthy thyroid counterparts. Of note, in the study by Zhu et al. only $30 \%$ of the investigated FTC tissue specimens and $20 \%$ of follicular adenomas stained positive for CXCR4 $4^{34}$. These discrepancies to our results may be explained by the small sample size investigated by Zhu et al. and the different dilution used for immunohistochemical staining. Nevertheless, our results fit perfectly into the accumulating literature, where in situ hybridization and immunohistochemistry analyses of different tumour tissue arrays have shown that the expression of both chemokine receptors is induced in various types of carcinomas ${ }^{36,37}$ and that it increases with malignancy ${ }^{13,15}$.

Fittingly, we found a strong association between high CXCR4 or CXCR7 expression and advanced tumour size as well as UICC stage. Additionally, both chemokine receptors demonstrated a strong negative association with patients' overall as well as recurrence free survival in the univariate analysis. In the multivariate analysis however, CXCR4 just missed statistical significance after the variable selection procedure based on the AIC. In the regression tree analysis on the other hand hierarchical clustering identified CXCR4 as the most powerful biomarker with the highest prognostic power in our set of patients. Even though the regression tree analysis is a solely exploratory statistical procedure, it still underlines the functional relevance of CXCR4 in FTC. These results are in line with the current literature, where recent studies have reported the prognostic relevance of CXCR4 and CXCR7 and their association with more advanced tumour stages in different tumour entities $37-39$. However, these findings have to be interpreted with care, as our sample size only allows limited propositions.

Considering their importance in the forming of distant metastases, the significantly higher expression of CXCR4 and CXCR7 in distant metastases compared to their expression in primary FTC specimens fit perfectly into our expanding knowledge of the
CXCR4/CXCR7/CXCL12 axis in carcinoma. Mechanistically, the homeostatic microenvironment chemokine CXCL12 functions as a chemo-attractant and cell activator ${ }^{40}$. Its binding to its respective receptors leads to the initiation of different cell signalling pathways, which in turn leads to the induction of EMT and the forming of an invasive phenotype $^{41-43}$.

In our functional in vitro experiments, we could demonstrate that both CXCR4 antagonizing compounds AMD3100 and WZ811 have a significant negative impact on the number of invading cells. Both compounds are known for their clinical potential in haematopoietic malignancies. While WZ811 significantly reduces $\mathrm{CD} 3+\mathrm{T}$ cell migration in vitro and in vivo, it also exercises strong anti-tumorigenic properties in chronic lymphocytic leukemia ${ }^{44,45}$. AMD3100 is mainly used in patients to facilitate the mobilization procedure of haematopoietic stem cells from the bone marrow. After bone marrow transplantation AMD3100 increases the number of circulating stem cells by inhibiting the CXCL12 mediated homing effect of the bone marrow microenvironment. This feature of AMD3100 modulated cell trafficking has been successfully implemented, especially in patients that failed to mobilize sufficiently after conventional medical procedures ${ }^{46,47}$. In addition, AMD3100 has been shown to supress tumour cell progression in small cell lung carcinoma cell lines ${ }^{48}$ and to encumber the extravasation process of metastasizing breast carcinoma cells ${ }^{49}$. Similar results have been reported on oral squamous cell carcinoma, where the CXCR4/CXCL12 axis was found to influence the formation of distant metastases 17,50 .

Of note, the co-incubation of FTC cells with rh-SDF1a and CXCR4-specific antagonists AMD3100 or WZ811 was not able to compensate the functional blockage of CXCR4. Since we could show that both chemokine receptors CXCR4 and CXCR7 are equally expressed in our cell model, this circumstance suggests that CXCR7 by itself is not capable of compensating a loss of function of CXCR4, implying CXCR4's functionality as a crucial requirement for CXCL12 mediated invasiveness in FTC. These observations are in line with reports that focus on CXCR7 ability to form functional heterodimers with CXCR4, thus acting more as a regulator of CXCR4 signalling than as an independent signal transducer ${ }^{15,51}$. Interestingly however, recent studies on PTC could demonstrate that CXCR7 by itself plays a crucial role regarding tumour progression, cell proliferation, angiogenesis and the forming of metastases both in vitro and in vivo $0^{32,33,52}$. Despite the fact that PTC and FTC both belong to the group of 
DTC, they both arise from different genetic backgrounds and offer profound genetic variations $^{53-55}$. Our findings therefore may just underscore one of these differences, further highlighting the complex interdependencies of the CXCR4/CXCR7/ CXCL12 axis in different entities. However, to fully elucidate the intricate interactions between CXCR4 and CXCR7 further functional studies will be required.

Rh-SDF1a induction of enhanced invasion was associated with changes in the expression of proteins involved in EMT. The expression level of E-cadherin decreased, whereas the expression of the mesenchymal marker, Vimentin, increased significantly after rh-SDF1a treatment ${ }^{56}$. CXCR4 had been previously associated with the induction of EMT in oral squamous cell carcinoma. Duan et al. could demonstrate that the silencing of CXCR4 was associated with a suppressed EMT and reduced tumour metastases ${ }^{57}$. CXCR7 on the other hand has been implicated in the promotion of EMT in lung cancer ${ }^{43}$.

In addition, the mRNA levels of EMT-associated transcriptional factors BST2, FGF9, SNAI1 and TWIST1 were markedly upregulated. BST2 was identified as an independent marker for metastasis in breast cell carcinoma ${ }^{58}$ and regulates EMT through activation of $\mathrm{NF}_{-\mathrm{K}} \mathrm{B}^{59,60}$. FGF9 has been linked to EMT through its upregulation of vascular endothelial growth factor ${ }^{61}$ and its expression has been associated with a metastatic phenotype in adenocarcinomas of the lung ${ }^{62}$. SNAI1 and TWIST1 on the other hand function synergistically as specific transcriptional repressors for the E-cadherin promoter63,64. Taken together, these changes in gene expression support the emerging picture of the CXCR4/CXCR7/CXCL12 axis as a key regulator of EMT.

FTC's largely beneficial course, even at a metastatic stage, is primarily based on adjuvant RAI treatment. However, once the tumour becomes refractory to RAI, tumour cell invasiveness and the forming of distant metastases determine the patient's outcome. With only limited success of current second line therapies, new insight into FTC's oncogenesis and invasive behaviour appears invaluable. Our results propose the CXCR4/CXCR7/CXCL12 axis as a leading functional aspect in FTC tumour cell invasiveness and possible therapeutic target. This valuable new perception should encourage future translational studies, to substantiate the chemokine receptors role in FTC tumour biology and confirm their worth as a part of a new multimodular therapeutic strategy.

\section{Abbreviations}

BST2: bone marrow stromal cell antigen 2; CI: confidence interval; CXCL12: C-X-C motif chemokine 12; CXCR4: C-X-C chemokine receptor type 4; CXCR7: C-X-C chemokine receptor type 7; DTC: differentiated thyroid carcinoma; EMT: epithelial-mesenchymal transition; FACS: fluorescence-activated cell sorting; FGF9: fibroblast growth factor 9; FTC: follicular thyroid carcinoma; HR: hazard ratio; IRS: immunoreactivity score; LND: lymph node dissection; ND: neck dissection; PTC: papillary thyroid carcinoma; RAI: radioactive iodine; REMARK: REporting recommendations for tumour MARKer prognostic studies; rh-SDF1a: recombinant human stromal cell-derived factor 1 a; SNAI1: Snail family transcriptional repressor 1; TWIST1: Twist homolog 1; UICC: Union internationale contre le cancer.

\section{Supplementary Material}

Supplementary table S1.

http:/ / www.jcancer.org/v09p0929s1.pdf

\section{Acknowledgements}

The study was supported, in part, by a grant from the Deutsche Forschungsgemeinschaft (KR 3496/2-1 to AK) and the Forschungskommission of the Medical Faculty, University Duesseldorf (39/2014 to AK and TAW)

\section{Competing Interests}

The authors have declared that no competing interest exists.

\section{References}

1. Dralle H, Musholt TJ, Schabram J, Steinmüller T, Frilling A, Simon D, Goretzki PE, Niederle B, Scheuba C, Clerici T, et al. German Association of Endocrine Surgeons practice guideline for the surgical management of malignant thyroid tumors. Langenbecks Arch Surg 2013; 398:347-75.

2. Haugen BR, Alexander EK, Bible KC, Doherty GM, Mandel SJ, Nikiforov YE, Pacini F, Randolph GW, Sawka AM, Schlumberger M, et al. 2015 American Thyroid Association Management Guidelines for Adult Patients with Thyroid Nodules and Differentiated Thyroid Cancer. Thyroid 2016; 26:1-133.

3. Asari R, Koperek O, Scheuba C, Riss P, Kaserer K, Hoffmann M, Niederle B. Follicular Thyroid Carcinoma in an Iodine-Replete Endemic Goiter Region. Ann Surg 2009; 249:1023-31

4. Gruber JJ, Colevas AD. Differentiated thyroid cancer: focus on emerging treatments for radioactive iodine-refractory patients. Oncologist 2015; 20:113-26.

5. Durante C, Haddy N, Baudin E, Leboulleux S, Hartl D, Travagli JP, Caillou B, Ricard M, Lumbroso JD, De Vathaire F, et al. Long-term outcome of 444 patients with distant metastases from papillary and follicular thyroid carcinoma: benefits and limits of radioiodine therapy. J Clin Endocrinol Metab 2006; 91:2892-9.

6. Anderson RT, Linnehan JE, Tongbram V, Keating K, Wirth LJ. Clinical, safety, and economic evidence in radioactive iodine-refractory differentiated thyroid cancer: a systematic literature review. Thyroid 2013; 23:392-407.

7. Vaisman F, Carvalho DP, Vaisman M. A new appraisal of iodine refractory thyroid cancer. Endocr Relat Cancer 2015; 22:R301-10.

8. Scala S. Molecular Pathways: Targeting the CXCR4-CXCL12 Axis--Untapped Potential in the Tumor Microenvironment. Clin Cancer Res 2015; 21:4278-85.

9. Balkwill F. Cancer and the chemokine network. Nat Rev Cancer 2004; 4:540-50.

10. Roland J, Murphy BJ, Ahr B, Robert-Hebmann V, Delauzun V, Nye KE, Devaux C, Biard-Piechaczyk M. Role of the intracellular domains of CXCR4 in SDF-1-mediated signaling. Blood 2003; 101:399-406.

11. Secchiero P, Celeghini C, Cutroneo G, Di Baldassarre A, Rana R, Zauli G. Differential effects of stromal derived factor-1a (SDF-1a) on early and late stages of human megakaryocytic development. The Anatomical Record 2000; 260:141-7.

12. Wright LM, Maloney W, Yu X, Kindle L, Collin-Osdoby P, Osdoby P. Stromal cell-derived factor- 1 binding to its chemokine receptor CXCR4 on precursor cells 
promotes the chemotactic recruitment, development and survival of human osteoclasts. Bone 2005; 36:840-53.

13. Sun X, Cheng G, Hao M, Zheng J, Zhou X, Zhang J, Taichman RS, Pienta KJ, Wang J. CXCL12 / CXCR4 / CXCR7 chemokine axis and cancer progression. Cancer Metastasis Rev 2010; 29.709-22.

14. Graham GJ. D6 and the atypical chemokine receptor family: Novel regulators of immune and inflammatory processes. European Journal of Immunology 2009; 39:342-51.

15. Sánchez-Martín L, Sánchez-Mateos P, Cabañas C. CXCR7 impact on CXCL12 biology and disease. Trends Mol Med 2013; 19:12-22.

16. Levoye A, Balabanian K, Baleux F, Bachelerie F, Lagane B. CXCR7 heterodimerizes with CXCR4 and regulates CXCL12-mediated G protein signaling. Blood 2009; 113:6085-93

17. Chen N, Jiang X, Wang J, Wu T, Cheng B, Xia J. CXCL12-CXCR4/CXCR7 axis contributes to cell motilities of oral squamous cell carcinoma. Tumor Biology 2015; 37:567-75.

18. Singh RK, Lokeshwar BL. The IL-8-Regulated Chemokine Receptor CXCR7 Stimulates EGFR Signaling to Promote Prostate Cancer Growth. Cancer Res 2011; 71:3268-77.

19. Würth R, Barbieri F, Bajetto A, Pattarozzi A, Gatti M, Porcile C, Zona G, Ravetti J-L, Spaziante R, Florio T. Expression of CXCR7 chemokine receptor in human meningioma cells and in intratumoral microvasculature. Journal of Neuroimmunology 2011; 234:115-23.

20. Brierley JD, Gospodarowicz MK, Wittekind C. TNM Classification of Malignant Tumours. John Wiley \& Sons; 2016.

21. Werner TA, Tamkan-Ölcek Y, Dizdar L, Riemer JC, Wolf A, Cupisti K, Verde PE, Knoefel WT, Krieg A. Survivin and XIAP: two valuable biomarkers in medullary thyroid carcinoma. Br J Cancer 2016; 114:427-34

22. Werner TA, Dizdar L, Nolten I, Riemer JC, Mersch S, Schütte SC, Driemel C, Verde PE, Raba K, Topp SA, et al. Survivin and XIAP - two potential biological targets in follicular thyroid carcinoma. Sci Rep 2017; 7:11383.

23. Remmele W, Hildebrand U, Hienz HA, Klein PJ, Vierbuchen M, Behnken LJ, Heicke B, Scheidt E. Comparative histological, histochemical, immunohistochemical and biochemical studies on oestrogen receptors, lectin receptors, and Barr bodies in human breast cancer. Virchows Arch A Pathol Anat Histopathol 1986; 409:127-47.

24. McShane LM, Altman DG, Sauerbrei W, Taube SE, Gion M, Clark GM, Statistics Subcommittee of the NCI-EORTC Working Group on Cancer Diagnostics. Reporting recommendations for tumor marker prognostic studies (REMARK). J. Natl. Cancer Inst.2005; 97:1180-4

25. Geldof AA, Versteegh LRT, van Mourik JC, Rooimans MA, Arwert F, Hermsen MA, Schadee-Eestermans IL, van Dongen GA, van der Valk P, van der Clement EHP, et al. Clonally related but phenotypically divergent human cancer cell lines derived from a single follicular thyroid cancer recurrence (TT2609). Thyroid 2001; 11:909-17.

26. Krieg A, Mersch S, Boeck I, Dizdar L, Weihe E, Hilal Z, Krausch M, Möhlendick B, Topp SA, Piekorz RP, et al. New model for gastroenteropancreatic large-cell neuroendocrine carcinoma: establishment of two clinically relevant cell lines. PLoS ONE 2014; 9:e88713.

27. Burger JA, Kipps TJ. CXCR4: a key receptor in the crosstalk between tumor cells and their microenvironment. Blood 2006; 107:1761-7.

28. Lagergren A, Månsson R, Zetterblad J, Smith E, Basta B, Bryder D, Akerblad P, Sigvardsson $\mathrm{M}$. The Cxcl12, periostin, and $\mathrm{Ccl} 9$ genes are direct targets for early B-cell factor in OP-9 stroma cells. Journal of Biological Chemistry 2007; 282:14454-62.

29. Livak KJ, Schmittgen TD. Analysis of relative gene expression data using real-time quantitative PCR and the 2(-Delta Delta C(T)) Method. Methods 2001; 25:402-8.

30. Burnham KP, Anderson DR. Model Selection and Multimodel Inference. Springer Science \& Business Media; 2003

31. Jung YH, Lee DY, Cha W, Kim BH, Sung M-W, Kim KH, Ahn S-H. Antitumor effect of CXCR4 antagonist AMD3100 on the tumorigenic cell line of BHP10-3 papillary thyroid cancer cells. Head Neck 2016; 38:1479-86.

32. Zhang H, Yang L, Teng X, Liu Z, Liu C, Zhang L, Liu Z. The chemokine receptor CXCR7 is a critical regulator for the tumorigenesis and development of papillary thyroid carcinoma by inducing angiogenesis in vitro and in vivo. Tumour Biol 2016; 37:2415-23.

33. Liu Z, Yang L, Teng X, Zhang H, Guan H. The involvement of CXCR7 in modulating the progression of papillary thyroid carcinoma. J Surg Res 2014; 191:379-88.

34. Zhu X, Bai Q, Lu Y, Lu Y, Zhu L, Zhou X, Wu L. Expression and function of CXCL12/CXCR4/CXCR7 in thyroid cancer. Int J Oncol 2016; 48.2321-9.

35. Liu Z, Sun D-X, Teng $X-Y, X u$ W-X, Meng X-P, Wang B-S. Expression of stromal cell-derived factor 1 and CXCR7 in papillary thyroid carcinoma. Endocr Pathol 2012; 23:247-53.

36. Gebauer F, Tachezy M, Zander H, Izbicki JR, Kaifi J, Bockhorn M. Prognostic Impact of CXCR4 And CXCR7 Expression In Pancreatic Adenocarcinoma. Journal of Surgical Research 2011; 165:176.

37. D'Alterio C, Consales C, Polimeno M, Franco R, Cindolo L, Portella L, Cioffi M, Calemma R, Marra L, Claudio L, et al. Concomitant CXCR4 and CXCR7 Expression Predicts Poor Prognosis in Renal Cancer. Current Cancer Drug Targets 2010; 10:772-81.

38. Schrevel M, Karim R, Haar ter NT, van der Burg SH, Trimbos JBMZ Fleuren GJ, Gorter A, Jordanova ES. CXCR7 expression is associated with disease-free and disease-specific survival in cervical cancer patients. Br J Cancer 2012; 106:1520-5.

39. Krieg A, Riemer JC, Telan LA, Gabbert HE, Knoefel WT. CXCR4--A Prognostic and Clinicopathological Biomarker for Pancreatic Ductal Adenocarcinoma: A Meta-Analysis. PLoS ONE 2015; 10:e0130192.

40. Mego M, Cholujova D, Minarik G, Sedlackova T, Gronesova P, Karaba M, Benca I, Cingelova S, Cierna Z, Manasova D, et al. CXCR4-SDF-1 interaction potentially mediates trafficking of circulating tumor cells in primary breast cancer. BMC Cancer 2016; 16:127.

41. Tu Z, Xie S, Xiong M, Liu Y, Yang X, Tembo KM, Huang J, Hu W, Huang X, Pan S, et al. CXCR4 is involved in CD133-induced EMT in non-small cell lung cancer. Int J Oncol 2016;
42. Sobolik T, Su Y-J, Wells S, Ayers GD, Cook RS, Richmond A. CXCR4 drives the metastatic phenotype in breast cancer through induction of CXCR2 and activation of MEK and PI3K pathways. Mol Biol Cell 2014; 25:566-82.

43. Wu Y-C, Tang S-J, Sun G-H, Sun K-H. CXCR7 mediates TGF $\beta 1$-promoted EMT and tumor-initiating features in lung cancer. Oncogene 2016; 35:2123-32.

44. Jung SH, Won KJ, Lee KP, Lee DH, Yu S, Lee D-Y, Seo E-H, Kang H, Park E-S, Kim $\mathrm{H}-\mathrm{J}$, et al. DJ-1 protein regulates CD3+ T cell migration via overexpression of CXCR4 receptor. Atherosclerosis 2014; 235:503-9.

45. Li SH, Dong WC, Fan L, Wang GS. Suppression of chronic lymphocytic leukemia progression by CXCR4 inhibitor WZ811. Am J Transl Res 2016; 8:3812-21.

46. Danylesko I, Sareli R, Varda-Bloom N, Yerushalmi R, Shem-Tov N, Shimoni A, Nagler A. Plerixafor (Mozobil): A Stem Cell-Mobilizing Agent for Transplantation in Lymphoma Patients Predicted to Be Poor Mobilizers - A Pilot Study. Acta Haematol 2016; 135:29-36.

47. Bilgin YM, de Greef GE. Plerixafor for stem cell mobilization: the current status. Curr Opin Hematol 2016; 23:67-71.

48. Taromi S, Kayser G, Catusse J, Elverfeldt von D, Reichardt W, Braun F, Weber WA, Zeiser R, Burger M. CXCR4 antagonists suppress small cell lung cancer progression. Oncotarget 2016; 7:85185-95.

49. Wendel C, Hemping-Bovenkerk A, Krasnyanska J, Mees ST, Kochetkova M, Stoeppeler S, Haier J. CXCR4/CXCL12 participate in extravasation of metastasizing breast cancer cells within the liver in a rat model. PLoS ONE 2012; 7:e30046.

50. Uchida D, Onoue T, Tomizuka Y, Begum NM, Miwa Y, Yoshida H, Sato M. Involvement of an autocrine stromal cell derived factor-1/CXCR4 system on the distant metastasis of human oral squamous cell carcinoma. Mol Cancer Res 2007; 5:685-94.

51. Sierro F, Biben C, Martinez-Munoz L, Mellado M, Ransohoff RM, Li M, Woehl B, Leung H, Groom J, Batten M, et al. Disrupted cardiac development but normal hematopoiesis in mice deficient in the second CXCL12/SDF-1 receptor, CXCR7. Proc Natl Acad Sci USA 2007; 104:14759-64.

52. Zhang $H$, Teng $X$, Liu $Z$, Zhang $L$, Liu $Z$. Gene expression profile analyze the molecular mechanism of CXCR7 regulating papillary thyroid carcinoma growth and metastasis. J Exp Clin Cancer Res 2015; 34:16.

53. Yoo S-K, Lee S, Kim S-J, Jee H-G, Kim B-A, Cho H, Song YS, Cho SW, Won J-K, Shin J-Y, et al. Comprehensive Analysis of the Transcriptional and Mutational Landscape of Follicular and Papillary Thyroid Cancers. PLoS Genet 2016; 12:e1006239.

54. Greco A, Borrello MG, Miranda C, Degl'Innocenti D, Pierotti MA. Molecular pathology of differentiated thyroid cancer. Q J Nucl Med Mol Imaging 2009; 53:440-53.

55. Rusinek D, Szpak-Ulczok S, Jarzab B. Gene expression profile of human thyroid cancer in relation to its mutational status. J Mol Endocrinol 2011; 47:R91-103.

56. Guarino M. Epithelial-mesenchymal transition and tumour invasion. Int J Biochem Cell Biol 2007; 39:2153-60.

57. Duan $Y$, Zhang S, Wang L, Zhou X, He Q, Liu S, Yue K, Wang X. Targeted silencing of CXCR4 inhibits epithelial-mesenchymal transition in oral squamous cell carcinoma. Oncol Lett 2016; 12:2055-61.

58. Woodman N, Pinder SE, Tajadura V, Le Bourhis X, Gillett C, Delannoy P, Burchell JM, Julien S. Two E-selectin ligands, BST-2 and LGALS3BP, predict metastasis and poor survival of ER-negative breast cancer. Int J Oncol 2016; 49:265-75.

59. Matsuda A, Suzuki Y, Honda G, Muramatsu S, Matsuzaki O, Nagano Y, Doi T, Shimotohno K, Harada T, Nishida E, et al. Large-scale identification and characterization of human genes that activate NF-kappaB and MAPK signaling pathways. Oncogene 2003; 22:3307-18.

60. Wu K, Bonavida B. The activated NF-kappaB-Snail-RKIP circuitry in cancer regulates both the metastatic cascade and resistance to apoptosis by cytotoxic drugs. Crit Rev Immunol 2009; 29:241-54.

61. Teishima J, Yano S, Shoji K, Hayashi T, Goto K, Kitano H, Oka K, Nagamatsu H, Matsubara A Accumulation of FGF9 in prostate cancer correlates with epithelial-to-mesenchymal transition and induction of VEGF-A expression. Anticancer Res 2014; 34:695-700

62. Ueng T-H, Chang Y-L, Tsai Y-Y, Su J-L, Chan P-K, Shih J-Y, Lee Y-C, Ma Y-C, Kuo M-L. Potential roles of fibroblast growth factor-9 in the benzo(a)pyrene-induced invasion in vitro and the metastasis of human lung adenocarcinoma. Arch Toxicol 2010; 84:651-60.

63. Feldmann G, Dhara S, Fendrich V, Bedja D, Beaty R, Mullendore M, Karikari C, Alvarez H, Iacobuzio-Donahue C, Jimeno A, et al. Blockade of hedgehog signaling inhibits pancreatic cancer invasion and metastases: a new paradigm for combination therapy in solid cancers. Cancer Res 2007; 67:2187-96.

64. Tomizawa Y, Wu T-T, Wang KK. Epithelial mesenchymal transition and cancer stem cells in esophageal adenocarcinoma originating from Barrett's esophagus. Oncol Lett 2012; 3:1059-63. 